\title{
Tecno-Estados, infoesfera y tecnopolítica ¿Cómo hackear las nubes?
}

Techno-States, infosphere and technopolitics How to hack the clouds?

Javier Echeverría

Ikerbasque

Lola S. Almendros

Instituto de Filosofía, CSIC

\section{RESUMEN}

Nuestra forma de vida está sufriendo un proceso de informatización que redefine las prácticas y relaciones de poder. En esta transformación, el Estado y el activismo tradicional han perdido capacidad de actuación y con ello poder. Para comprender esta pérdida y desarrollar nuevos modos de resistencia, es necesario entender el modo en que están configuradas las relaciones de poder: qué es y cómo se hace la política en la actualidad.

La filosofía de la información de Luciano Floridi ofrece un buen marco conceptual para caracterizar esta nueva forma de vida, pero considera el proceso de informatización como una cuestión ontológica que conduce a una visión del ecosistema informacional como un entorno neutral. Esto obstaculiza la capacidad crítica de una forma de vida cuya lógica simbiótica pone en jaque la libertad. Nuestra intención es completar sus análisis desde una perspectiva axiológica y política, con el fin de caracterizar un conjunto de condiciones para el desarrollo de resistencia política efectiva en la era informacional.

PALABRAS CLAVE: política, valores, filosofía de la información, agencia, hacktivismo 


\begin{abstract}
Our way of life is going through a process of informatization that redefines practices and power relations. In this transformation, the State and traditional activism have lost their capacity to act and, thus, their power. To realize this situation, and to be able to develop new modes of resistance, it is necessary to understand how power relations are being configured: what politics is and how it is done today.

Luciano Floridi's philosophy of information offers a good conceptual framework to characterize this new way of life. But he considers the process of computerization as an ontological issue, which leads to an informational ecosystem vision as neutral environment. This hinders the critical capacity in a way of life whose logic is symbiotic and compromises freedom. Our intention is to complete his analysis from an axiological and political perspective, in order to characterize a set of conditions for the development of effective political resistance in the informational age.
\end{abstract}

KEY WORDS: politics, values, philosophy of information, agency, hacktivism

\title{
INTRODUCCIÓN
}

La revolución tecnocientífica transformó radicalmente la práctica científica durante el siglo XX. Su carácter innovador tuvo efectos disruptivos no solo en múltiples ámbitos científicos y tecnológicos, también sociales; y sobre todo en la génesis de nuevos sectores económicos basados en la información (data economy o knowledge economy). El programa NBIC (nano-bio-info-cogno) de la National Science Foundation (NSF) ha aportado, durante más de una década, una main stream a la tecnociencia del siglo XXI, que sigue plenamente activa a finales de 2018, y con tendencia a crecer. Diversos subprogramas derivados del NBIC, como las Industrias 4.0, el análisis de big data, los programas BRAIN y HUMAN BRAIN, la Inteligencia Artificial (deep learning y redes neuronales convolucionales), la Technological Singularity, o el blockchain están produciendo transformaciones mucho más profundas que las ya causadas por las tecnologías de la información y la comunicación (TICs) y las biotecnologías desde finales del siglo XX. En lo que respecta al carácter político de las sociedades tecnocientíficas, las redes sociales son el elemento más innovador, no solo porque son la principal fuente de información, sino por su incidencia en los modos de hacer y (re)presentar la política. 
La revolución tecnocientífica ha cambiado nuestras sociedades de manera sustancial, sobre todo tras el giro informacional. A lo largo de este proceso, las Ciencias Sociales y las Humanidades normalmente han ido a remolque, cuando no se han opuesto a ella. Sin éxito, en ambos casos. Así, perduran claramente marginadas, con excepción de aquellos grupos que han evolucionado hacia lo tecnosocial y lo tecnohumano actuando habitualmente como meros comentaristas o futurólogos, bien colmados de optimismo, bien catastrofistas, pero en todo caso poco ilustrados y aún menos críticos. Los transhumanistas son representantes claros de esta transición, y no tardarán mucho en aparecer también nichos de "trans-sociales".

Nuestra contribución aquí se centrará ante todo en la aparición de la infoesfera (Floridi) y la tecnopolítica, cuyos efectos están siendo devastadores para los agentes, prácticas y sistemas políticos surgidos en los siglos XIX y XX. Cierto es que dichos sistemas políticos, los Estados-Nación con democracias liberales más o menos socialdemócratas, para entendernos, no han desaparecido. Pero su crisis es profunda y su influencia menguante, sobre todo a escala global, donde priman claramente los poderes tecno-financieros y tecno-militares. Estos nuevos tecnopoderes neoliberales están modificando el sentido, el valor y las posibilidades de los Estados y las Naciones.

La tecnopolítica está caracterizada por el sistema de acciones políticas que, para ser llevadas a cabo, requieren necesariamente de instrumentos, mediaciones y conocimientos tecnológico-informacionales. Para clarificar la cuestión, consideremos la deuda pública de los Estados, la gestión de sus Haciendas y fondos de bienestar, las negociaciones presupuestarias, o la gestión de los gobiernos y administraciones, que se basan y justifican en datos e información. Otro tanto sucede con las campañas electorales, que se hacen sobre todo en las redes sociales, siendo cada vez más residuales los escenarios clásicos de acción política (casas de gobierno, parlamentos, tribunales de justicia, debates entre líderes de los partidos, manifestaciones en calles y plazas, barricadas, huelgas generales, etc.). Sin duda, todas estas cuestiones son políticas, pero también son tecnológicas. La mediación tecno- ha ido instaurándose progresivamente en las acciones de los tres poderes del Estado, informatizándolos. Este fenómeno ha sido mucho más intenso y profundo en relación con el "Cuarto Poder".

Los poderes globales financieros, informacionales y comunicacionales determinan cada vez más las agendas y acciones políticas, incluidas las que tienen que ver con la participación de la ciudadanía, que hoy en día es, ante todo, tecno-participación a través de las redes sociales y las corrientes de tecno- 
opinión gestionadas por empresas. Toda la "cadena de valor" de los "mercados políticos" está mediatizada por sistemas tecnológicos cada vez más complejos, sofisticados, incontrolables y difíciles de regular. Este panorama obliga a distinguir las prácticas políticas tradicionales de las nuevas prácticas tecnopolíticas.

Qué es y cómo se hace la política en la era de la información son las preocupaciones que impulsan nuestra investigación. Comprender esta circunstancia es imprescindible para definir nuevas formas de resistencia sociopolítica. Nuestro objetivo es definir el tipo de resistencia política que podría ser exitosa en la actualidad, esto es, efectiva, trascendente y, sobre todo, disruptiva. Ahora bien, para entender las nuevas prácticas en general y las políticas en particular, hay que entender la tecnología. En este sentido la filosofía política tiene que ser filosofía de la información, pues la información misma - y no simplemente su uso -, se ha convertido en algo no solo político - como ya bien indicó Langdon Winner $(1980,1989)$ a propósito de la tecnología en general - sino tremendamente poderoso. Analizar esta metamorfosis es esencial para el objetivo de este trabajo.

La filosofía de la información de Luciano Floridi ofrece herramientas conceptuales que presentan un fuerte valor epistémico para nuestro fin, pero no termina de explicar el carácter político de la información. Si bien sus tesis constituyen gran parte del marco teórico de referencia, nuestra intención es complementarlas desde una perspectiva propia de los Estudios Sociales de la Ciencia y la Tecnología. Así, para elaborar una caracterización que permita comprender el significado político actual de nuestro entorno e (inter)acciones, en primer lugar, analizaremos las virtudes y limitaciones de la propuesta de Floridi, prestando especial atención a sus inconvenientes ontológicos y epistémicos. En segundo lugar, se analizarán los tres conceptos con que el filósofo italiano define el entorno, los sujetos y la forma de vida: infoesfera, inforg y onlife (Floridi, 2015). La infoesfera supone una representación de la realidad como (eco)sistema informacional, donde la información es estructura y relación, forma y contenido... en definitiva: Todo. El concepto inforg denota una redefinición de la idea de sujeto y agencia que conlleva la formulación de una antropología que no es antropológica ni antropocéntrica, e incita a una revisión radical de la ética. Por último, onlife indica la superación de la dicotomía onlineoffline, definiendo así el modo de vida simbiótico propio del ecosistema informacional. Explicaremos por qué esta forma de vida pone en jaque cuestiones de alta relevancia política como la libertad. 
Este recorrido por la cartografía del mundo y las relaciones informacionales pondrá de relieve que la filosofía de la información de Floridi acusa un reduccionismo ontológico: la topología de la infoesfera no contempla valores ni intereses, por ello deja de lado lo político. Sin axiología, la infoesfera toma la apariencia de un espacio estructural y relacionalmente neutral. Además, la concentración del filósofo italiano en la agencia como categoría ética central, limita la reflexión en términos conductistas, pues incita a pensar que los conflictos en los procesos informacionales dependen solo (o al menos fundamentalmente) de las actuaciones. El dinamismo de la infoesfera se asocia y restringe de este modo a la agencia, y no se consideran las constricciones para el funcionamiento, ejercicio y desarrollo de las capacidades que posibilitan la agencia misma. Nosotros, en cambio, sostendremos que tales constricciones explican las formas de poder en la actualidad. Así, nuestro análisis político de la infoesfera parte de la idea de que no es un ecosistema neutral: la información tiene valor, se valora, genera y destruye valor. Complementar la filosofía de la información de Floridi desde una perspectiva axiológica es fundamental para comprender el significado de lo político en nuestros días.

Para finalizar, atenderemos a los efectos de esta circunstancia en la génesis y desarrollo de mecanismos de resistencia política. Se expondrán las razones por las que los ciberactivismos, al dirigirse a los Estados, no son efectivos, y se explicará por qué la resistencia política tiene que adoptar formas hacker.

\section{¿POR QUÉ LA FILOSOFÍA DE LA INFORMACIÓN ES FILOSOFÍA POLÍTICA?}

¿Cómo hacer política si no hay alternativa? ¿Y cuando las alternativas están en las nubes? Estas dos preguntas marcan la historia del neoliberalismo, desde La Dama de Hierro a Jeff Bezos. El panorama nuboso parece avecinar democracia. Las BigTech han dividido el cielo a golpe de innovación y con San Pedro en paro por fin podemos elegir donde esperar el chaparrón. Comprender y hacer política hoy pasa por entender las tecnologías y saber actuar con ellas.

El giro computacional supuso la implementación de lo digital en nuestras vidas. En este sentido podemos caracterizarlo como el efecto vital de los primeros desarrollos tecnocientíficos en el ámbito de las ciencias computacionales. Este giro se define por el avance técnico. El giro informacional, en cambio, es el resultado del perfeccionamiento del sistema tecnocientífico. No se caracteriza por el avance técnico sino por el (socio)económico. Por ello su impacto en la forma de vida es mucho mayor, de modo que nuestra imagen del mundo está siendo modificada, al igual que nuestras prácticas, capacidades y posibilidades. 
En este sentido, el giro informacional no solo representa un cambio de paradigma porque ofrece una nueva realidad que comprender, sino también una nueva realidad que configurar. Así, aunque el giro informacional es una cuestión epistémica, fundamentalmente es un asunto político.

La información es el elemento clave para la comprensión del alcance del giro informacional, pero no es una cuestión sencilla. La información no solo estructura las prácticas, también la comprensión de las prácticas, por eso tiene (o adopta) múltiples sentidos, significados y valores, llegando así a ser el sustrato ontológico de la filosofía de la información de Luciano Floridi. Ahora bien, la omnipresencia de la información hace que sea, ante todo, poder.

Aunque el giro computacional ya supuso la digitalización de muchas de nuestras prácticas y relaciones, fue conservador. El giro informacional, sin embargo, está siendo disruptivo. El cambio que evidencia la ruptura de paradigma comienza en la concepción de la información, pues a sus características cuantitativas y cuantificables, construidas y explotadas desde la época computacional, se ha añadido un inconmensurable valor cualitativo, también cuantificable, y por tanto explotable, además, de diversas maneras.

El desarrollo, difusión y uso de tecnologías digitales conformó nuevos tipos de prácticas y relaciones entre las cosas, entre las personas, y entre las personas y las cosas. El giro informacional supone la redefinición de las prácticas y relaciones de y entre las cosas, de y entre las personas, y de y entre las cosas y las personas. $\mathrm{Y}$ aunque dichas relaciones mantienen su sentido computacional, su estructura es híper-conexionista, de modo que es mucho más complejo de-limitar no solo las entidades sino las acciones mismas. Este modo relacional extensivo dinamiza (y acelera) las praxis, pero dificulta su comprensión. Además, este éxito funcional implica una personificación de las cosas que nace de la confusión de la autonomía con el automatismo. Esta tendencia se sigue de la irrupción de la agencia en las cosas: tendemos a creer que las cosas, al ser automáticas, son autónomas. Al mismo tiempo, al tomar como norma el automatismo de la agencia de las cosas, el comportamiento de las personas se acaba por pensar automatizado. La informatización de las prácticas humanas supone su automatización, poniendo así en peligro su autonomía. De este modo, en el otro lado de la moneda, encontramos la correspondiente cosificación de las personas, cuyos estragos peligrosamente se diluyen pues al fin y al cabo la híperconectividad es efectiva. Luciano Floridi explica este éxito funcional en términos ontológico-informacionales desde una perspectiva realista-estructuralista. Nosotros, en cambio, creemos que es una cuestión política. 
La aparición de nuevos modos de comprender y conceptualizar la realidad es un síntoma habitual de un paradigma en quiebra. Por ello Floridi sostiene que su filosofía de la información puede adoptarse como filosofía primera. En esta filosofía primera la información es la esencia estructural y relacional de la realidad - de la infoesfera -. La infoesfera no es el ciberespacio, tampoco es un entorno virtual soportado por el mundo material genuino (Floridi 2010a). Es " the whole international environment constituted by all informational entities (thus including informational agents as well), their properties, interactions, processes, and mutual relations" (Floridi 2007: 59). Esto significa que en la infoesfera no hay entidades que no sean informacionales. En este sentido la filosofía de la información consiste en una labor de re-conceptualización y también de reontologización, pues convierte en información a las cosas, personas, y (la estructura de) relaciones. Aunque la postura de Floridi tiende a ser kantiana, el peso de su categorización ontológica eclipsa la componente epistemológica: la información es fenómeno pero también es noúmeno. No solo la comprensión de la realidad estaría informatizada, la realidad misma sería información. Si bien esta aseveración sirve para cerrar la argumentación que constituye el marco teórico floridiano, presenta problemas de justificación, además de regatear la crítica acerca de la información. Si la información es el elemento esencial, "lo dado", es fácil tomarla como sustrato neutral. Consecuencia de ello, Floridi centra las cuestiones éticas en los agentes y en los usos de la información, pasando desapercibida la relevancia de su carga axiológica y política.

Nuestra postura respecto de la información es considerablemente distinta. En primer lugar, si la información es todo, eso significa que puede ser cualquier cosa, por lo que no resulta muy útil como recurso explicativo (a no ser que se pretenda hacer metafísica). En segundo lugar, que el mundo funcione informacionalmente no implica necesariamente que sea informacional. En tercer lugar, que la construcción de conocimiento acerca de la realidad esté informatizada - que conceptualizar la realidad hoy pase por informatizarla -, no significa que la realidad sea información. El problema, por tanto, no es si todo es información, sino que la información está en todo. Su omnipresencia le otorga omnipotencia, al tiempo que dificulta la capacidad de comprender su poder para generar entonces algún tipo de resistencia.

No es que se haya descubierto que la naturaleza es algorítmica, más bien porque las (inter)acciones, decisiones y prácticas se han informatizado, también lo han hecho las posibilidades de entender el mundo, y de actuar en él. Es la lógica de nuestro tiempo, pues establece su ritmo y reglas. Ahora bien, esto no se debe a que el mundo sea (o no) informacional como sugiere Floridi, sino a que opera de 
manera informacional. Esta cuestión no es ontológica. La información es ecosistema, pero no solo en sentido epistémico, pues también lo es en sentido práctico (y por ello político).

En definitiva, el ecosistema informacional, la infoesfera, no es algo dado: se ha constituido y permanece, cambia y se conserva. Hay que comprender cómo y por qué sucede esto. Atender a los agentes no es muy provechoso, pues es difícil detectarlos y peligroso reducirlos a su función. Si adoptamos un marco de reflexión sistémico estamos bien situados para conceptualizar esta operatividad conexionista. Pero esta virtud epistémica se pierde si deriva en lo ontológico, esto es, en la reducción de la explicación a una "naturaleza" informacional. La infoesfera es más bien un reino nuboso que, emergido desde el "tercer entorno" (Echeverría 1999), transfigura las relaciones y el poder del resto de entornos.

\section{LAS CARACTERÍSTICAS POLÍTICAS DE LA VIDA ONLIFE}

El giro computacional mantuvo una distinción bastante clara entre lo digital y lo analógico. También era fácil diferenciar lo online y lo offline. La intrusión (no demasiado intensa ni extensa) de sistemas de computación y conexión no adulteró la lógica mecánica basada en la sucesión lineal causa-efecto con la que se pensaba el mundo. Además, siguió vigente el ideario de progreso moderno asociado al control, el avance y la mejora. $\mathrm{Y}$ aun rodeada de expectativas en el desarrollo tecnocientífico que aludían a lo político, la categoría de sujeto preservó su solidez psicológica, jurídica y ética. En esta época también apareció la metáfora del ciborg como símbolo (tecno)político de emancipación (Haraway 1991). De todo ello poco queda.

La lógica mecánica lineal no es aplicable a una estructura (eco)sistémica. Tampoco tiene sentido asociar progreso y control al conocimiento gestado desde el aparataje tecnocientífico. La innovación en tecnociencia ha supuesto una brecha de la que han emergido nuevos significados, valores y poderes para $-\mathrm{y}$ desde - la información. El modelo computacional tampoco es suficiente: la híper-conectividad tiene lugar en términos más complejos. No es que sea más difícil distinguir lo analógico de lo digital, tampoco es que se haya digitalizado lo analógico, más bien ya no existe lo uno y lo otro. La indefinición de lo online y lo offline es causa (y consecuencia) de un nuevo modo de estar y actuar en el mundo: el modo onlife. Floridi explica este nuevo modo existencial en términos informacionales, biológicos y simbióticos. Esto otorga consistencia a su idea de ecosistema informacional, pues implica, además de la informatización de la 
estructura del ecosistema, también la de las relaciones que lo constituyen y mantienen. La infoesfera sería de este modo un (eco)sistema autoregulado.

$\mathrm{Al}$ reconocer que la simbiosis informacional propia de la vida onlife conlleva modificaciones en la idea de sujeto y de agencia, el filósofo italiano desarrolla una ética basada en una "mindless morality, distributed agency and moraly, deantropocentric and de-antropological idea of action" (Floridi, 2013a: 261). Los agentes son así el centro gravitatorio, "[...] which also include non-human (artificial) and non-individual (distributed) entities, as well as networked, multiagent systems and hybrid agents (e.g. companies and institutions)" (Floridi 2009b: 650). Al ser la agencia el elemento cardinal, la ética floridiana es, además de contextualista y consecuencialista, fundamentalmente conductista:

[moral actions present the following components:] agent, patient, interactions between them, agent's general frame of information, factual information concerning the situation (part avaible to the agent), general environment (where agent and patient are located), and specific situation in which interactions occurs (Floridi 2013b: 103).

$\mathrm{Al}$ mismo tiempo, el papel ético de los inforgs es considerablemente kantiano, pues presentan valor moral intrínseco: "All entities, when interpreted as clusters of information - when our ontology is developed at an informational level of abstraction - have a minimal moral worth qua informational entities and so may deserve to be respected" (Ibíd.: 102). Esta categoría se debe al foco en la agencia. Para Floridi, los inforgs son agentes morales, poiéticos (2010b), pues actúan en el (eco)sistema y lo modifican. De ello que la tónica consecuencialista de su ética se configure desde la idea de entropía (Floridi 2013b: 159): el (des)orden del infoesfera - las consecuencias de las acciones - sería el indicador de la moralidad de las acciones.

La vida onlife es la vida de los inforgs. "informational organisms (...), mutually connected and embedded in an informational environment (infosphere), which we shave with other informational agents, both natural and artificial, that also process information logically and autonomously" (Floridi 2014: 94). Donde "autonomía" poco tiene que ver con su pasado moderno-liberal, pues, como se ha visto, más bien implica automatismo: esto es, osmosis para la simbiosis. La filosofía de la información conduce así a una antropología que no es ni antropológica ni antropocéntrica. Floridi describe este cambio como una cuestión emergentista: "Inforg" denota "[the] emergence of artificial and hybrid 
(multi)agents, that is partly artificial and partly human" (2007: 62). La hibridación no sería protésica sino natural, ecológica más bien.

Los inforgs están integrados prácticamente en el ecosistema informacional; carecen de carácter unitario pues son parte indiferenciada del sistema. El sujeto se convierte así en un sistema distribuido y dinámico, pero ¿ello supone el fin del sujeto, de su unidad e identidad? Al responder a estas cuestiones el filósofo italiano "naturaliza" su ontologización informacional. Para ello, postula la existencia de tres membranas de naturaleza informacional (corporal, cognitiva y consciente) cuya interacción tiene lugar de manera homeostática. Así se explicarían los procesos y relaciones sistémicas propias de los inforgs al tiempo que mantendrían su carácter unitario e identitario aun formando parte de la infoesfera. Esta estructura y mantenimiento del inforg - su existencia espacial y temporal -, se soporta en el intercambio de información entre las membranas, y entre estas y el medio. Ahora bien, considerar que las relaciones simbióticas de los inforgs y la infoesfera se deben a que estos son entidades informacionales implica, además del reduccionismo ontológico ya criticado, un determinismo que esconde las causas y efectos políticos de dichas relaciones. La simbiosis no es ontológica sino funcional. No se debe a que seamos información sino a que estamos obligados a operar informacionalmente: la información (se) crea y (se) destruye, por eso, (nos) transforma.

Las metáforas computacionales y cibernéticas que sirvieron para conformar la idea de ciborg no dan cuenta del alcance del giro informacional. No todos los inforgs son humanos y solo algunos humanos son inforgs. Los artefactos, maquinales o algorítmicos, que también entran en la definición de Floridi, aunque operan solos pertenecen a quienes acceden a "simbiotizarse" más profundamente. Este acceso está mediado por una de las pocas cosas que sigue operando con fuerte valía desde el segundo entorno: el dinero. Formar parte del ecosistema informacional de una manera profunda requiere artefactos, pues son los que potencian la agencia. Otra cosa es que dicha agencia sirva para hacer algo realmente.

El inforg es un constructo, no un descubrimiento. No son seres informacionales, son el resultado de informatizar la categoría de sujeto y agencia, esto es, de informatizar los modos de subjetivación y las praxis. En definitiva, son el resultado de una exigencia simbiótica fruto las relaciones de poder que han construido la forma de vida actual. Esta pérdida de identidad, capacidad y agencia es absolutamente democrática, pues nos afecta a todos. Participar en esta forma de vida implica una cesión de libertad de tal calibre que hablar de 
desigualdad empieza a carecer de sentido. Por muy compleja que sea la infoesfera no deja de ser un entorno nuboso bien configurado y mantenido desde el monopolio BigTech.

El inforg no es consecuencia de la naturaleza informacional de humanos y cosas. Más bien simboliza la informatización a la que hoy se ve sometida cualquier cosa. Es el modo de llamar al resultado de un proceso de des-subjetivación que ha personificado las cosas y ha cosificado a las personas. La relación simbiótica de los inforgs en, con y desde la infoesfera representa un modo de alienación. Del mismo modo que la infoesfera es el resultado de la expansión del "tercer entorno", este modo de subjetivación es el resultado de las relaciones de poder establecidas e impuestas desde este no-lugar. La conectividad simbiótica de la infoesfera se soporta en la ambigüedad de las nubes, donde operan bien coordinadas la incapacidad de definir la información, su propiedad y su aprovechamiento. En este sentido la infoesfera no solo no es neutral, tampoco es democrática.

Los simbiontes informacionales ya no son (ni están) sujetos, pero ¿es posible la emancipación de un simbionte? Esta cuestión es fundamental para comprender la resistencia política en la actualidad.

\section{(TECNO)RESISTENCIA Y HACKTIVISMO}

La idea moderna de Estado se sustenta en el imaginario del progreso. El poder se entiende asociado a la soberanía, lo cual implica capacidad de elección, decisión y acción, o lo que es lo mismo, de control. Las razones fundamentales para la existencia del Estado son la libertad y la igualdad, y la democracia es el modo en que estas (en el mejor de los casos) progresan desde la soberanía. La lógica rectora es también moderna, mecánica: unos agentes-representantes, unas circunstancias, una toma de decisiones para cambiarlas, y unos cambios efectivos, que más o menos son los previstos. El Estado es regulación porque se basa en la predicción. Pero el mundo del progreso, la soberanía y la previsión, no es el del ecosistema informacional. Creer que es así supone caer en una visión solucionista de la tecnología (Morozov 2012, 2013) que hace pasar una circunstancia de caos bien confeccionada (y beneficiosa para unos pocos) por una apertura perenne de posibilidades de progreso. No olvidemos que la infoesfera es fruto de la innovación que, a diferencia del progreso, implica incertidumbre y no control: la imposibilidad de predecir y por ello de regular (y mucho menos de solucionar). Por ello la tecnopolítica no puede ser 
parlamentaria, aunque sí debería poder ser democrática. Ahora bien, como veremos, esta cuestión no solo es política, también es epistémica.

¿Cómo se ha colado la lógica de la innovación en lo político? El neoliberalismo es la respuesta. Al menos cuatro factores han sido clave en este proceso. En primer lugar, la ausencia de regulación del despliegue económico en el plano financiero-informacional. En segundo lugar, la emergencia de un mercado informacional, donde la información es mercancía y capital. Lo que ha provocado un cambio disruptivo en la cadena de valor. En tercer lugar, la génesis de valor económico de la información a partir de su valor social y político. Esto es, una subversión del modo de concretarse la cadena de valor. Y en último lugar, el fin de la distinción entre lo público y lo privado que se ha consolidado como consecuencia de la privatización de lo público que marca la decadencia del estado de bienestar, y de la publicación de lo privado que sostiene las comunicaciones que plagan todo espacio y todo tiempo.

Desde el "tercer entorno", los señores de las nubes no solo han expoliado el poder político y el social, se nutren de la explotación de escándalos políticos y sociales tan efímeros como paralizadores. Lo sociopolítico es un catalizador que sirve para que el ruido del malestar hinche las nubes. De este modo se ha generado una estructura de poder paralela con un alto impacto sociopolítico que consiste en dejar las cosas como están y que, al mismo tiempo, parezca que no hay alternativa. Sus efectos, además de ser difícilmente previsibles - y por tanto regulables -, no son ni abarcables ni gestionables por los Estados, cuyo papel en esta transformación, en el mejor de los casos, ha sido pasivo (recuérdese el fracaso de las Cumbres Mundiales sobre la Sociedad de la Información, organizadas por la ONU en 2003 y 2005). La conjunción de esta permisividad con el rápido desarrollo de innovaciones en lo económico y lo tecnológico ha abierto una brecha irreparable en las formas de poder, entre las que el Estado se mantiene a modo de parafernalia. Esta transformación de las relaciones de poder no solo ha saltado fronteras, las ha disuelto. El resultado es un mapa geopolítico donde los Estados como entidad jurídico-política tienen poco terreno y cada vez menos competencia. Tampoco las grandes uniones estatales están representando una mayor capacidad de actuación. De los déficits en lo estatal y la incompetencia de las colaboraciones internacionales surge el exacerbo de lo nacional para todos los gustos que se ha puesto de moda en los últimos tiempos. Las naciones tampoco son ya un espacio identitario, de comunidad, por eso lo mejor que ofrece la democracia de partidos, a uno y otro lado del Atlántico, es populismo. 
A estos problemas se suma la decadencia material de los Estados en forma de deuda pública, y una incapacidad fáctica para la actuación. Lo único que la democracia representativa (re)presenta hoy es espectáculo. Esta tragicomedia apunta a una falsa confianza de "reír por no llorar". Además, ninguno de los pocos intentos de innovación política se ha desligado de la lógica partidista, y a falta de poder (hacer) a lo sumo llegan a ser tweets.

La innovación en el plano económico ha consistido en una redescripción de la cadena de valor, donde lo fundamental ha dejado de ser la relación entre producción de bienes y su consumo, pasando a ser el modelo de venta y el cliente. Con el auge de las TICs la distancia entre cliente y vendedor se ha acortado, y no solo en tiempo y espacio. La exposición y flujo contantes de información permiten una personalización de la venta de elevada eficiente y eficaz. La lógica se ha invertido: no está primero el producto (que es lo valioso) y luego el consumo, sino el estudio del cliente para determinar qué venderle. El modelo de negocio de las redes sociales se basa en el aprovechamiento económico (en la génesis de valor) de la informatización de lo social. En este sentido, lo social ha sido reformulado en términos de capital. Esta informatización de lo social (y su consiguiente mercantilización) supone una fuente de poder de crecimiento exponencial. La fórmula mágica del neoliberalismo consiste precisamente en aliviar los síntomas de esta circunstancia, pues explota la voluntad de cesión de libertad haciendo que la subordinación pase por la máxima expresión de la libertad misma: haciéndonos simbiontes. Esta circunstancia paradójica se sustenta en un aparente vacío de poder que se sigue de las dificultades para su detección. Por ello el poder en la era informacional es un poder nuboso. Entre dichas dificultades destaca, por un lado, lo bien camuflados que están los nodos de poder, y por otro lado que el elemento de coacción - el elemento "disciplinario" -, es la libertad misma. La evolución del neoliberalismo ha consistido en un proceso contradictorio, extremadamente funcional - y por ello exitoso -, en el que lo económico se ha instalado en la información y la información ha logrado valor económico a costa de lo social. Que el valor económico se geste a expensas de lo social supone un modo de poder de carácter político ejercido desde el plano económico. De ello la ausencia de neutralidad del ecosistema informacional.

El valor económico de la información genera disvalor político porque pone en jaque la libertad. Al exponer información privada (de manera manifiesta o inconsciente) con cada una de nuestras interacciones donde están presentes instancias informacionales (que son mercantiles) perdemos libertad, pues facilitamos las posibilidades de manipulación. El origen del valor económico de 
la información está en su uso para perfeccionar las técnicas de marketing y publicidad, lo que incide en nuestro comportamiento. La creación de valor económico a través de la información privada y la información derivada de las interacciones mediadas por instancias informacionales supone una fuente de poder cuyas consecuencias políticas, dada esta afectación de la libertad, no son positivas. En este sentido, la filosofía de la información es filosofía política no solo porque la información es política sino porque es un problema político.

Las TICs en general y las redes sociales en particular tienen un protagonismo especial en esta metamorfosis de la forma de vida, pues han sido fundamentales en la informatización de toda práctica y la mercantilización de toda información. Por ello Floridi no las define como "technologies of the self", sino como "egopoietic technologies" (Floridi 2011a: 155). Configuran un proceso de subjetivación que ya no es foucaultiano $(2000,2002)$. Tampoco es una cuestión de sutileza y eficiencia psicopolítica, tal como viene indicando Byung-Chul Han (2012, 2013, 2014a, 2014b). Es un proceso mucho más complejo, en el que se mantiene la identidad a la par que se distribuye, y se promueve la agencia al tiempo que se supedita a las condiciones contractuales impuestas por los propietarios de las nubes para formar parte de un entramado social que es mercantil. Así se exalta el ejercicio de una libertad bien circunscrita. No conviene entender este proceso de subjetivación como una circunstancia ontológica, hacerlo así implica no solo aceptar la lógica neoliberal sino promover su inocencia. Estamos ante una innovación en la subjetivación. La innovación en la concreción de la cadena de valor en el plano económico neoliberal se ha trasladado al ámbito sociopolítico en los mismos términos. En los ciberespacios sociales no somos ciudadanos, ni siquiera usuarios, somos productores y consumidores.

El Estado ha perdido poder, por lo que no tiene sentido el desarrollo de una resistencia política que le interpele: el fin del poder del Estado implica el fin del activismo en términos tradicionales. El ciberactivismo ha reproducido las formas y prácticas del viejo activismo, por eso no funciona. Además, lo ha hecho desde "las nubes". Esto es, desde plataformas emergidas de la explotación mercantil de la informatización de las relaciones sociales. Las redes sociales que difunden sus discursos y posibilitan su organización impiden la trascendencia de sus reivindicaciones y actuaciones. La híperinformación desvirtúa el sentido y significado de los contenidos, obligando a apadrinar las arengas de postverdad. La información se crea y se difunde, tiene valor, se valora, genera y destruye valor. El carácter viral de unas cuestiones y no otras, y la ambigüedad que arremete contra el valor epistémico de la verdad nutren un ecosistema en el que 
lo que se dice se convierte en ruido, y lo que se hace en fotogramas. Los usuarios (al tiempo que clientes) y productores (al tiempo que consumidores), participan del espectáculo político como extras de una narración sin trama. Las reivindicaciones no solo se "quedan en las nubes", las alimentan. Y es que, aunque lo de Cambridge Analytics nos atormente, solo es la punta del iceberg.

En las redes sociales la individuación máxima se confunde con la máxima expresión de la individualidad. La informatización de las relaciones sociales que está en la base del valor económico de la información tiene entre sus consecuencias una homogeneización de las identidades (individuales y colectivas), que supone la pérdida de su significado político en favor de su explotación económica. Esta híper-conexión homogeneizante genera también una falsa idea de igualdad que arremete contra la capacidad de detectar instancias de reconocimiento $\mathrm{y}$, por tanto, de detectar problemas de subordinación de estatus.

En definitiva, la falta de trascendencia de los ciberactivismos se debe, por un lado, a que sus propósitos y modos de operar siguen anclados en la distinción online-offline. Las ciberplataformas sociales, en el mejor de los casos, sirven para la comunicación, discusión y organización. Las reivindicaciones y la acción, en cambio, están dirigidas al mundo offline. Además, su interlocutor no ostenta el poder, pues continúa siendo el Estado. Por otro lado, si bien las plataformas favorecen cuestiones como la capacidad de organización y visibilidad, el trazo de estrategias, o la captación de adeptos, al mismo tiempo clausuran - o directamente censuran - la crítica y actividad realmente subversivas. En este sentido, la propia estructura que sirve de organización a los colectivos activistas es la que impide su proyección política. El altruismo "democrático" de estos espacios eclipsa los focos de poder. La benevolencia de las plataformas sociales permite el ruido de las quejas al tiempo que las comercializa: no solo las ensordece, las convierte en moda. Por ello el ataque a las plataformas que están modificando la cadena de valor social y económico es el único que puede resultar transformador.

\section{CONCLUSIONES}

No es la información lo que estructura la infoesfera, es la informatización de la forma de vida lo que nos obliga a una existencia simbionte donde lo social y lo económico se funden acabando con lo político. El poder de la información nace de la informatización de toda circunstancia y posibilidad, determinando las prácticas y anulando así su capacidad subversiva. Sin lugar a duda, la 
informatización de la forma de vida ha sido una innovación disruptiva. Recuperar la libertad pasa por recuperar la agencia, lo que necesariamente implica atentar contra el ecosistema informacional. La (tecno)resistencia no puede tener lugar desde las nubes sino contra las nubes, por eso tiene que ser hacker.

Como se ha visto, la condición simbiótica de los inforgs no es ontológica sino práctica. El inforg es actor, por eso se cree libre. Ahora bien, su margen de actuación está bien delimitado, por eso su hiperactividad no tiene efectos disruptivos. Las formas de poder se sustentan en las prácticas, por lo que la resistencia también ha de ser cuestión de prácticas, concretamente, de conocimiento práctico. Y es que para dar respuesta a la pregunta sobre cómo emancipar un simbionte hay al menos cuatro cuestiones fundamentales que considerar, donde lo epistémico y lo político se entremezclan:

1. Comprensión de la circunstancia simbionte.

2. Comprensión de las condiciones de la simbiosis.

3. Comprensión de la lógica de la simbiosis.

4. Capacidad de atentar contra la lógica de la simbiosis.

Las tres primeras condiciones requieren el desarrollo de investigaciones sociales, económicas y políticas. La última exige el desarrollo de conocimiento técnico impregnado de valores subversivos cuya concreción depende de las condiciones previas. Para cumplir con las exigencias de comprensión de las primeras condiciones es necesario, en primer lugar, el desarrollo de un estudio profundo de la economía informacional, en el que se preste especial atención a las características, causas y efectos, de las nuevas cadenas de valor desde una perspectiva que aborde el mercado como proceso y no como estructura, pues la lógica que impera es la de la innovación (ver Calderini, Garrone y Sobrero 2003; Schumpeter 2003; Aghion y Banerjee 2005). En segundo lugar, hay que estudiar, por un lado, los mecanismos de mercadotecnia que se dirigen a la comprensión y manipulación del comportamiento de los individuos y, por otro lado, los mecanismos técnicos de expolio y explotación de la información, desde el análisis de big data, la data mining, y el machine learning, a los algoritmos que estructuran la web 2.0, o los que dotan a las cosas de "inteligencia". En tercer lugar, conviene relacionar cada uno de estos mecanismos con las empresas BigTech que los desarrollan y utilizan, sus fines explícitos e implícitos y sus posibles consecuencias. Esta cuestión es fundamental para entender cómo se consolidan los monopolios de las nubes. 
En cuarto lugar, hay que crear un marco de comprensión antropológico, social y político que explique las características de la forma de vida onlife, que profundice en las razones por las cuales la voluntad de los individuos sostiene las relaciones informacionales, y que atienda a las consecuencias y la proyección que tiene todo ello para la convivencia social y política. En quinto lugar, es necesario definir y contextualizar los problemas políticos propios de la vida onlife, así como los valores implicados. Entre estos destacan valores como la transparencia - con su efecto en cuestiones como la intimidad -, pero también otros como el compartir, cuyo sentido ha sido dislocado. Indagar la significación de los valores puede aportar indicios para transgredir o reinventar sus significados. Por ello, en sexto lugar, la descripción de los valores operantes tiene que ir acompañada de una propuesta de valores que subvierta el orden establecido. Entre estos habría de estar la anonimidad contra la transparencia, el compartir cifrado contra el compartir abierto, la creación contra el uso y el consumo, la conexión independiente y elegida contra la conexión dependiente y subordinada, y sobre todo la sorpresa y el ataque contra la estrategia y la pasividad. Así, en último lugar, es importante confeccionar prácticas que materialicen tales valores y configuren subjetividades que subviertan el exacerbo de la individualidad en pro de la concreción de colectivos con problemas y objetivos comunes.

En lo que respecta a la cuarta condición - a la capacidad de atentar contra la lógica de la simbiosis -, lo que está en juego es la posibilidad de emprender acciones empoderadoras. Para ello es imprescindible contar con un alto conocimiento técnico que pueda ser utilizado contra la estructura (de poder) informacional que sostiene la vida onlife. Este conocimiento técnico tiene que suponer una creación destructiva, por ello debe nutrirse de los frutos de las tareas asociadas a las tres primeras condiciones.

En definitiva, nuestro trabajo ha pretendido mostrar las características de este totalitarismo de la información, poniendo de relieve sus estragos políticos. La información tiene valor $\mathrm{y}$ se valora, genera y destruye valor. La (tecno)resistencia tiene que alterar dicha valor-acción. Puesto que el poder de las nubes es difícilmente identificable, los colectivos hacktivistas tampoco pueden ser identificables si se persigue el empoderamiento. Tampoco puede serlo su actividad: deben ser comunidades plásticas, nubosas, de ataque imprevisible, tormentosas. La anonimidad es la condición política para el ejercicio de la libertad onlife. 


\section{BIBLIOGRAFÍA}

AGHION, P. y BANERJEE, A. (2005): Volatility and Growth, Oxford (UK), Oxford University Press.

CALDERINI, M., GARRONE, P. y SOBRERO, M. (2003): Corporate Governance, Market Structure and Innovation, Cheltenham (UK), Edwar Elgar.

ECHEVERRÍA, J. (1999): Los señores del aire: Telépolis y el tercer entorno, Barcelona, Destino.

FLORIDI, L. (2007): "A Look into the Future Impact of ITC on Our Live", The Information Society, 23, 1, pp. 59-64. Consultado: 20 de septiembre 2018 (https://doi.org/10.1080/01972240601059094).

FLORIDI, L. (2009a): “Against Digital Ontology”, Synthese, 168, pp. 151-178. Consultado: 20 de septiembre 2018 (https://doi.org/10.1007/s1129-008-93346).

FLORIDI, L. (2009b): "Network Ethics: Information and Business Ethics in a Networked Society", Journal of Business Ethics, 90, pp. 649-659. Consultado: 20 de septiembre 2018 (https://doi.org/10.1007/s10551-010-0598-7).

FLORIDI, L. (2010a): Ethics After the Information Revolution, en The Cambridge Handbook of Information and Computer Ethics, editado por L. Floridi, New York, Cambridge University Press, pp. 3-19.

FLORIDI, L. (2010b): The Ethics of the Information Society in a Globalized World, en The Cambridge Handbook of Information and Computer Ethics, editado por L. Floridi, New York, Cambridge University Press, pp. 271-283.

FLORIDI, L. (2010c): Information. A Very Short Introduction, New York, Oxford University Press.

FLORIDI, L. (2011a): "The Informational Nature of Personal Identity", Mind \& Machines, 21, pp. 549-566. Consultado: 20 de septiembre 2018 (https://doi.org/10.1007/s11023-011-9259-6).

FLORIDI, L. (2011b): The Philosophy of Information, New York, Oxford University Press.

FLORIDI, L. (2012): “Turing's Three Philosophical Lessons and the Philosophy of Information", Philosophical Transactions of Royal Society A, 370, pp. 3536-3542. Consultado: 20 de septiembre 2018 (https://doi.org/10.1098/rsta.2011.0325). 
FLORIDI, L. (2013a): "Distributed Morality in an Information Society", Science and Engineering Ethics, 19, pp. 727-743. Consultado: 20 de septiembre 2018 (https://doi.org/10.1007/s11948-012-9413-4).

FLORIDI, L. (2013b): The Ethics of Information, Oxford, Oxford University Press.

FLORIDI, L. (2014): The Fourth Revolution. How the Infosphere is Reshaping Human Reality, Oxford, Oxford University Press.

FLORIDI, L. (ed.) (2015): The Onlife Manifesto. Being Human in a Hyperconnected Era. SpringerOpen. Consultado: 20 de septiembre 2018 (https://doi.org/10.1007/978-3-319-04093-6).

FOUCAULT, M. (2000): Vigilar y castigar: el nacimiento de la prisión, México, Siglo XXI.

FOUCAULT, M. (2002): La hermenéutica del sujeto: Curso en el Collège de France (1981-1982), México, Fondo de Cultura Económica.

HAN, B. (2012): La sociedad del cansancio, Barcelona, Herder.

HAN, B. (2013): La sociedad de la transparencia, Barcelona, Herder.

HAN, B. (2014a): En el enjambre, Barcelona, Herder.

HAN, B. (2014b): Psicopolítica. Neoliberalismo y nuevas técnicas de poder, Barcelona, Herder.

HARAWAY, D. (1991): Simians, Cyborgs, and Women. The Reinvention of Nature, New York, Routledge.

MOROZOV, E. (2012): El desengaño de Internet: los mitos de la libertad en la red, Barcelona, Destino.

MOROZOV, E. (2013): To Save Everything, Click Here: The Folly of Technological Solutionism, New York, PublicAffairs.

SCHUMPETER, J. (2003): Capitalism, Socialism and Democracy, New York, Reoutledge.

WINNER, L. (1980): “Do Artifacts Have Politics?', Daedalus, 109, 1, pp. 121-136. Consultado: 26 de septiembre de 2018 (http://www.jstor.org/stable/20024652).

WINNER, L. (1989): The Whale and the Reactor. A Search for Limits in an Age of Hight Technology, Chicago, University of Chicago Press. 
Javier Echeverría Ezponda es miembro Jakiunde, Academia de las Ciencias, de las Artes y de las Letras del País Vasco. Sus principales campos de investigación han sido la filosofía de la ciencia y la tecnología; la ética de la ciencia; el estudio de las relaciones entre ciencia, tecnología y sociedad, las nuevas tecnologías de la información y la comunicación y los estudios de innovación. Entre sus libros publicados en los últimos años destacan: Telépolis (1994); Cosmopolitas Domésticos (1995); Los Señores del Aire: Telépolis y el Tercer Entorno (1999); Introducción a la Metodología de la Ciencia: la Filosofía de la Ciencia en el siglo XX (1999); Ciencia y Valores (2002); La revolución tecnocientífica (2003), Gobernar los riesgos: Ciencia y valores en la sociedad del riesgo (edición en colaboración con J. L. Luján, 2004), Ciencia del bien y el mal (2007) y La luz de la luciérnaga. Diálogos de Innovación Social (con Ander Gurrutxaga, 2012). Es miembro de la International Academy of the Philosophy of Science y vicepresidente de la Sociedad Española Leibniz. Ha recibido el Premio Anagrama de Ensayo (1995), el Premio Euskadi de Investigación en Humanidades y Ciencias Sociales (1997), el Premio Nacional de Ensayo (2000), otorgado por el Ministerio de Cultura por su obra Los Señores del Aire y el Premio Eusko Ikaskuntza-Laboral Kutxa (2016).

Lola S. Almendros es investigadora predoctoral en el Departamento de Ciencia, Tecnología y Sociedad del IFS-CSIC (España). Su línea de investigación aborda las consecuencias sociopolíticas del establecimiento de la ideología de la transparencia, los problemas jurídico-políticos y de privacidad presentes en el desarrollo de las redes sociales y las posibilidades de desarrollos tecnopolíticos de las prácticas democráticas y feministas en los entornos sociales digitales. Entre sus publicaciones, se encuentran "De la sociedad de la información a la sociedad del conocimiento", "Technoscience and Democracy: Epistemic and Political Problems and Open Movements in the Attachment of Knowledge Societies" e "Ingenierías, sociedades digitales e infoesfera: una interpretación de la filosofía y la ética de la información de Luciano Floridi” junto a Javier Echeverría Ezponda. 